\title{
Anaphylaxis and Undiagnosed Aspirin Exacerbated Respiratory Disease in the Ambulatory Surgery Center: A Case Report
}

\author{
Jennifer Wu MD \\ Department of Anesthesiology, University of Texas Health Science Center Houston, Houston, Texas \\ Email: JenniferWuMD@gmail.com
}

Received 12 November 2015; accepted 19 December 2015; published 22 December 2015

Copyright (C) 2015 by author and Scientific Research Publishing Inc.

This work is licensed under the Creative Commons Attribution International License (CC BY).

http://creativecommons.org/licenses/by/4.0/

(c) (i) Open Access

\begin{abstract}
Severe bronchospasm and anaphylaxis are unanticipated emergencies that may occur in the ambulatory surgery setting. I present a case in which an asthmatic male with nasal congestion has anaphylaxis after induction, with severe bronchospasm as the primary manifestation. During the course of hospitalization, he was exposed to aspirin and a second episode of severe bronchospasm occurred. He was diagnosed with both anaphylaxis to an anesthetic medication and Aspirin Exacerbated Respiratory Disease, or Samter's Triad.
\end{abstract}

\section{Keywords}

Ambulatory Anesthesia, Bronchospasm, Anaphylaxis, Anaphylactoid, Samter's Triad, Aspirin Exacerbated Respiratory Disease

\section{Introduction}

Severe allergic reactions occur in the perioperative setting in approximately 1:10,000 to 1:20,000 cases [1] [2]. These can occur unexpectedly in the absence of history or prior exposure. Signs and symptoms include urticaria, pruritis, nasal congestion, lacrimation, wheezing, bronchospasm, angioedema, tachycardia and hypotension. Treatment of severe allergic reactions includes epinephrine and supportive care. Anaphylaxis and anaphylactoid reactions in the ambulatory surgery setting are of concern because the invasive supportive care, pressors, and prolonged ventilation may not be available. Stabilization and swift transfer to a hospital is critical. Transfer will also allow the patient to be monitored in a hospital in the event of recrudescence, which may occur in up to $20 \%$ of cases [3]. This article reports a case in which a patient with undiagnosed Aspirin Exacerbated Respiratory Disease go into severe bronchospasm following induction and intubation at an ambulatory surgery center, and 
again the following day after receiving aspirin in the ICU.

\section{Case Description}

A 39-year-old male presented to the Ambulatory Surgery Center (ASC) on the day of surgery for elective endoscopic sinus surgery. He received phone screening by an ASC nurse a few days prior. The nurse anesthetist and anesthesiologist performed the preoperative assessment on the day of surgery. His medical history was for significant for asthma, nasal congestion, and environmental allergies. He had been diagnosed with asthma a year prior after an exposure to environmental allergens caused wheezing. In the past year, he had intermittently used his albuterol inhaler up to five times daily, but often went for weeks without symptoms. The patient never had anesthesia and there was no family history of general anesthesia complications.

On the day of surgery, his lungs were clear to auscultation bilaterally. His airway exam revealed Mallampati I, good mouth opening, and normal neck mobility. He was complaining of nasal congestion.

In the preoperative holding area, the patient took two puffs of his albuterol inhaler. He received midazolam 2 $\mathrm{mg}$, ranitidine $20 \mathrm{mg}$, and ondansetron $4 \mathrm{mg}$ IV. In the operating room, standard ASA monitors were placed and the patient was preoxygenated. During induction, the patient received fentanyl $100 \mathrm{mcg}$, lidocaine $100 \mathrm{mg}$, and propofol $200 \mathrm{mg}$ IV. Once easy mask ventilation was established with oxygen and sevoflurane, the patient received rocuronium $40 \mathrm{mg}$ IV. Of note, the patient did not receive preoperative antibiotics. Direct laryngoscopy revealed a grade I view and a lidocaine LTA was used prior to easily placing an endotracheal tube.

Following placement of the endotracheal tube, no condensation was noted in the tube, no end tidal $\mathrm{CO}_{2}$ was obtained, and there were no breath sounds. The patient was difficult to bag ventilate. Once endotracheal tube placement was confirmed, bronchospasm was suspected. Ventilation attempts with oxygen and sevoflurane were continued and albuterol was administered via an MDI adapter. Despite these efforts, the patient was still not being ventilated. Epinephrine $100 \mathrm{mcg}$ IV was administered and a tidal volume of $100 \mathrm{ml}$ was achieved. The patient also received diphenhydramine $50 \mathrm{mg}$ and hydrocortisone $100 \mathrm{mg}$ IV. Hypoventilation persisted and epinephrine was continuously administered to achieve tidal volumes of $100-200 \mathrm{ml}$. The case was cancelled and the decision was made to transfer the patient to a hospital. During this time, the patient received an additional 10 $\mathrm{mg}$ of rocuronium to avoid movement during transport. The patient continued to require incremental epinephrine IV to maintain ventilation.

Upon arrival to the Emergency Department of a nearby hospital, the patient began to exhibit signs of hemodynamic instability. He was initially in sinus tachycardia with frequent PVCs with a rate up to $180 \mathrm{bpm}$. He quickly became bradycardic at $30 \mathrm{bpm}$ and hypotensive at $60 / 40 \mathrm{~mm} \mathrm{Hg}$. Abdominal ultrasound revealed a flat inferior vena cava, suggesting vasodilation or hypovolemia. Treatment with epinephrine IV, atropine IV, and intravenous fluid boluses restored his heart rate and blood pressure. He remained intubated and ventilation improved during this time. He was admitted to the Cardiac Intensive Care Unit (ICU) and extubated seven hours after the episode of bronchospasm. He remained in the Cardiac ICU for observation. Because of his episodes of tachycardia and bradycardia, cardiac enzymes were drawn and his highest troponin level was $2.33 \mathrm{ng} / \mathrm{ml}$. As per Cardiac ICU protocol, the patient received aspirin as treatment for a positive troponin. One hour after receiving the aspirin, which was seventeen hours after the initial episode of bronchospasm, the patient again went into bronchospasm. He was reintubated with proprofol and succinylcholine. Bronchoscopy was within normal limits. He improved and was extubated twelve hours later.

The patient was diagnosed with an anaphylactic reaction to an induction agent and Samter's Triad. He was discharged the following day (post-operative day two) with instructions to seek allergy testing and management of his asthma.

\section{Discussion}

The patient described in this case report suffered from severe bronchospasm, most likely caused by allergic reaction to an anesthetic agent that was administered preoperatively or at induction. Anaphylaxis is an immunoglobulin (Ig) E mediated severe reaction to an agent that occurs following at least one initial exposure to the antigenic agent. A non-IgE mediated, or anaphylactoid, reaction occurs when direct activation of mast cells causes the release of inflammatory mediators and no prior exposure is necessary [4]. Both anaphylaxis and the anaphylactoid reaction can present with urticaria, pruritis, nasal congestion, lacrimation, wheezing, bronchospasm, angioedema, and hemodynamic instability. In patients with underlying asthma, the initial presentation of 
an allergic reaction may be severe bronchospasm. Anaphylactic and anaphylatoid reactions have the same presentation and are undistinguishable by serum triptase level. The most common triggers of severe perioperative allergic reaction are nondepolarizing muscle relaxants, latex, and antibiotics [5]. Anesthesiologists should provide a letter to patients who suffer from a severe reaction and should include the medications administered, a description of the reaction, and response to treatment. Patients can provide this information to an allergist for testing and also to their surgeon and anesthesiologist for any future procedures so that a second severe reaction may be avoided.

Following removal of the suspected antigen, the most important medication to treat the severe allergic reaction is epinephrine. Prompt administration of epinephrine will stabilize the mast cell to halt the release of histamine and other inflammatory mediators. The action of epinephrine at the beta 2 receptor will cause bronchodilation and help counteract wheezing and bronchospasm. The vascular effects will correct the hypotension and also help to counter the compensatory tachycardia [6] [7]. Other management includes diphenhydramine, albuterol, intravenous fluid, and steroids. Supportive care may include additional vascular access, invasive arterial and central venous pressure monitoring, pressors, and ventilation with an endotracheal tube.

In addition to the intraoperative reaction, this patient suffered from a severe reaction to aspirin. Based on the symptoms, timing of the aspirin administration, and past medical history, he was diagnosed with Samter's Triad, or Aspirin Exacerbated Respiratory Disease (AERD).

AERD is a triad of nasal polyps or congestion, asthma, and aspirin sensitivity. The prevalence is about $6 \%$ in adults, but up to $26 \%$ in patients with both nasal polyps and asthma. Patients with this condition often undergo multiple sinus surgeries [8]. Anesthesiologists must be aware of this condition, particularly in the population undergoing sinus surgery. One should carefully consider avoiding the use of ketorolac and other nonsteroidal anti-inflammatory drugs (NSAIDs) in this population. In addition, some patients with AERD have had reactions to high dose (>1000 mg) intravenous acetaminophen [9]. A careful history must be obtained to guide post-operative pain management.

\section{Funding}

None.

\section{Conflicts of Interest}

None.

\section{References}

[1] Levy, J.H. and Adkinson Jr., N.F. (2008) Anaphylaxis during Cardiac Surgery: Implications for Clinicians. Anesthesia \& Analgesi, 106, 392-403. http://dx.doi.org/10.1213/ane.0b013e3181602e0d

[2] Mertes, P.M. and Laxenaire, M.C. (2004) Allergy and Anaphylaxis in Anaethesia. Minerva Anestesiologica, 70, 285-291.

[3] Johnson, R.F. and Peebles, R.S. (2004) Anaphylactic Shock: Pathophysiology, Recognition, and Treatment. Seminars in Respiratory and Critical Care Medicine, 25, 695-703. http://dx.doi.org/10.1055/s-2004-860983

[4] Peavy, R.D. and Metcalfe, D.D. (2008) Understanding the Mechanisms of Anaphylaxis. Current Opinion in Allergy \& Clinical Immunology, 8, 310-314. http://dx.doi.org/10.1097/ACI.0b013e3283036a90

[5] Hepner, D.L. and Castells, M.C. (2003) Anaphylaxis during the Perioperative Period. Anesthesia \& Analgesi, 97, 1381-1395. http://dx.doi.org/10.1213/01.ANE.0000082993.84883.7D

[6] Dewachter, P., Mouton-Faivre, C. and Emala, C.W. (2009) Anaphylaxis and Anesthesia: Controversies and New Insights. Anesthesiology, 111, 1141-1150. http://dx.doi.org/10.1097/ALN.0b013e3181bbd443

[7] Dewachter, P., Mouton-Faivre, C., Emala, C.W., Beloucif, S. and Riou, B. (2011) Case Scenario: Bronchospasm during Anesthetic Induction. Anesthesiology, 114, 1200-1210. http://dx.doi.org/10.1097/ALN.0b013e3182172cd3

[8] Kim, J.E. and Kountakis, S.E. (2007) The Prevalence of Samter’s Triad in Patients Undergoing Functional Endoscopic sinus Surgery. Ear, Nose \& Throat Journal, 86, 396-399.

[9] Settipane, R.A., Schrank, P.J., Simon, R.A., Mathison, D.A., Christiansen, S.C. and Stevenson, D.D. (1995) Prevalence of Cross-Sensitivity with Acetaminophen in Aspirin-Sensitive Asthmatic. Journal of Allergy and Clinical Immunology, 96, 480-485. http://dx.doi.org/10.1016/S0091-6749(95)70290-3 